\title{
Etude des phases d'un clinker par diffractométrie des rayons $X$ : vers la quantification
}

\author{
M. Signes-Frehel, P. Maes et C. Haehnel \\ Centre Technique, Groupe ITALCEMENTI-CIMENTS Français, Rue des Technodes, 78931 Guerville \\ cedex, France
}

\begin{abstract}
:
In the cement industry clinker, a synthetic material obtained from clay and limestone, is the result of a complex industrial process. The nature of the resulting phases and their relative proportions in the clinker are essential elements for a clear understanding of its chemical and mechanical properties.

Selective dissolution methods enable the mineralogical phases to be isolated.

They are analysed by diffraction and the measurement of the relative areas of the characterstic peaks of the phases of the clinker, with no internal standard, enables the information obtained to be related to the expected properties of the final product.

This method of identifying the composition of the clinker permits a refinement of the results obtained by $\mathrm{X}$-ray fluorescence.
\end{abstract}

\section{Résumé :}

Dans l'industrie cimentière, le clinker, matériau noble par excellence, obtenu à partir d'argile et de calcaire, est le résultat d'un processus industriel complexe. La nature des phases qui en résultent et leurs proportions relatives dans le clinker sont les éléments essentiels d'une bonne compréhension des propriétés chimiques et mécaniques du ciment.

Les méthodes de dissolution sélective permettent d'isoler les phases minéralogiques.

Celles-ci sont analysées par diffraction et la mesure des aires relatives des pics caractéristiques des phases du clinker, sans étalon interne, conduit à relier les informations obtenues aux propriétés que l'on attend du produit final.

La composition du clinker ainsi obtenue permet d'affiner les résultats fournis par la fluorescence X.

\section{Généralités :}

Le ciment est un liant hydraulique, c'est-à-dire qu'il forme avec l'eau une pâte plastique «faisant prise et durcissant progressivement, même à l'abri de l'air, notamment sous l'eau» (définition de la norme française NF P 15-301).

Le processus de fabrication suit le schéma général suivant :

- Extraction des matières premières,

- Broyage et homogénéisation du cru,

- Cuisson, obtention du clinker,

- Broyage du clinker additionné de gypse et obtention du produit final : le ciment.

\section{Exploitation de la carrière}

De celle-ci on tirera les matières premières nécessaires à la constitution d'un mélange finement divisé et homogénéisé appelé cru constitué pour l'essentiel de calcaire (carbonate de calcium) et d'argile (silice, alumine et oxyde de fer). 
Les proportions des oxydes entrant dans la composition du cru répondent à des exigences rigoureuses, de sorte qu'il est parfois nécessaire d'ajuster la composition du mélange par incorporation de minéraux extérieurs au site exploité comme de la pyrite grillée pour l'apport de fer, de sable pour la silice ou de calcaire pour la chaux.

Après broyage et homogénéisation, la composition moyenne d'un cru se situe dans les limites et les moyennes fournies dans le tableau ci-dessous :

\begin{tabular}{|l|l|l|}
\hline OXYDES & LIMITES (\%) & VALEUR MOYENNE (\%) \\
\hline $\mathrm{CaO}$ & $60-69$ & 65 \\
$\mathrm{SiO}_{2}$ & $18-24$ & 21 \\
$\mathrm{Al}_{2} \mathrm{O}_{3}$ & $4-8$ & 6 \\
$\mathrm{Fe}_{2} \mathrm{O}_{3}$ & $1-8$ & 3 \\
$\mathrm{MgO}$ & $<5$ & 2 \\
$\mathrm{~K}_{2} \mathrm{O}, \mathrm{Na}_{2} \mathrm{O}$ & $<2$ & 1 \\
$\mathrm{SO}_{3}$ & $<3$ & 1 \\
\hline
\end{tabular}

\section{Clinkérisation}

Le cru ainsi préparé appelé encore farine subit un traitement thermique comprenant un préchauffage nécessaire à l'évaporation de l'eau superficielle et à la dissociation de l'eau liée chimiquement. La décarbonatation démarrée lors de l'étape décrite précédemment est poursuivie par passage dans un échangeur à cyclones pour subir une précalcination afin d'accroître le taux de décarbonatation de la farine de l'ordre de $30 \%$ avant son entrée dans le four.

La progression de la farine dans le four avec élévation de température entre 1000 et $1450^{\circ} \mathrm{C}$, donne lieu à la formation d'une phase pâteuse dans laquelle les différentes espèces minérales se combinent pour donner un nouveau produit de synthèse : le clinker. A la sortie du four, le clinker subit une trempe, déterminante pour les propriétés physiques que l'on exige du produit final : le ciment, obtenu par incorporation de gypse au clinker finement broyé.

La composition chimique du clinker est facilement obtenue par fluorescence $\mathrm{X}$ et donne pour un clinker Portland [*] la composition suivante :

\begin{tabular}{|l|l|l|}
\hline OXYDES & NOTATION CIMENTIERE & $\%$ \\
\hline $\mathrm{SiO}_{2}$ & $\mathrm{~S}$ & 19 à 25 \\
$\mathrm{Al}_{2} \mathrm{O}_{3}$ & $\mathrm{~A}$ & 2 à 8 \\
$\mathrm{CaO}$ & $\mathrm{C}$ & $62-67$ \\
$\mathrm{Fe}_{2} \mathrm{O}_{3}+\mathrm{FeO}$ & $\mathrm{F}$ & 1 à 5 \\
$\mathrm{MgO}$ & 0 à 3 \\
$\mathrm{Na}_{2} \mathrm{O}+\mathrm{K}_{2} \mathrm{O}$ & & 0.2 à 1.3 \\
\hline
\end{tabular}

La composition des clinkers et des ciments peut être évaluée par des ratios calculés ou modules qui tiennent uniquement compte des éléments majeurs. Les plus utilisés sont le facteur de saturation en chaux noté $\mathrm{K}$, le module silicique ou MS et le module aluminoferrique $\mathrm{A} / \mathrm{F}$. Ils permettent de préparer et comparer facilement les clinkers. Parmi les formules servant à calculer la teneur en composants cristallins 
d'après la composition chimique, la formule de BOGUE (1929) donne, à titre d'exemple, pour un clinker normal les relations suivantes:

$\begin{array}{lll}\mathrm{C}_{3} \mathrm{~S} & =4.071 \mathrm{C}-7.602 \mathrm{~S}-6.719 \mathrm{~A}-1.430 \mathrm{~F} \\ \mathrm{C}_{2} \mathrm{~S} & =8.602 \mathrm{~S}+5.068 \mathrm{~A}+1.079 \mathrm{~F}-3.070 \mathrm{C} \\ & \text { ou } & 2.868 \mathrm{~S}-0.754 \mathrm{C}_{3} \mathrm{~S} \\ \mathrm{C}_{3} \mathrm{~A} & =2.650 \mathrm{~A}-1.692 \mathrm{~F} \\ \mathrm{C}_{4} \mathrm{AF} & =3.043 \mathrm{~F}\end{array}$

Dans ces conditions, $l^{1}$ histoire thermique joue en grand rôle dans les formes cristallines et minéralogiques des phases présentes en proportions variables :

\begin{tabular}{|l|l|l|l|}
\hline $\begin{array}{l}\text { NOTATION } \\
\text { CIMENTIERE }\end{array}$ & NOMS & $\begin{array}{l}\text { FORMULE } \\
\text { CHIMQQUE }\end{array}$ & TENEUR \% \\
\hline $\mathrm{C}_{3} \mathrm{~S}$ & Silicate tricalcique ou alite & $3 \mathrm{CaO}, \mathrm{SiO}_{2}$ & $40<60<70$ \\
$\mathrm{C}_{2} \mathrm{~S}$ & Silicate bicalcique ou bélite & $2 \mathrm{CaO}, \mathrm{SiO}_{2}$ & $0<15<30$ \\
$\mathrm{C}_{3} \mathrm{~A}$ & Aluminate tricalcique & $3 \mathrm{CaO}, \mathrm{Al}_{2} \mathrm{O}_{3}$ & $2<8<15$ \\
$\mathrm{C}_{4} \mathrm{AF}$ & Alumino-ferrite tétracalcique & $4 \mathrm{CaO}, \mathrm{Al}_{2} \mathrm{O}_{3}, \mathrm{Fe}_{2} \mathrm{O}_{3}$ & $0<8<15$ \\
\hline
\end{tabular}

En effet, la nature du combustible (fuel, coke de pétrole, charbon, ...) et le pouvoir oxydant ou réducteur de la flamme modifient les constituants du clinker qui ne sont pas des composés purs, mais des solutions solides contenant des impuretés dans leur réseau cristallin(1). Dans des teneurs variant du pourcent pour les plus abondantes à quelques ppm, les impuretés principales des différentes phases se répartissent globalement comme suit :
Alite
$: \mathrm{Fe}, \mathrm{Al}, \mathrm{Mg}, \mathrm{S}$
Bélite
: Fe, Al, P, S, K
Aluminate tricalcique
: $\mathrm{Si}, \mathrm{Mg}, \mathrm{Ti}, \mathrm{Cr}, \mathrm{Mn}$
et contribuent à modifier la réactivité du clinker.

[*] Nom du lieu où les matériaux de composition chimique idéale ont été trouvés à l'état naturel.

\section{Apport de la diffraction}

A partir de ces données générales, les techniques de diffraction des rayons $\mathrm{X}$ vont permettre de préciser la nature des phases présentes dans le clinker et en donner une évaluation quantitative. Cependant, la tâche est rendue délicate dans la mesure où les minéraux de clinker présentent de nombreuses formes allotropiques. Ainsi le silicate tricalcique $\left(\mathrm{C}_{3} \mathrm{~S}\right)$ offre six formes allotropiques depuis la température ambiante jusqu'à $1100^{\circ} \mathrm{C}$ alors qu'il en existe cinq entre la température ambiante et $1500^{\circ} \mathrm{C}$ pour le silicate bicalcique $\left(\mathrm{C}_{2} \mathrm{~S}\right)$. Si l'aluminate tricalcique $\left(\mathrm{C}_{3} \mathrm{~A}\right)$ ne présente pas de transformation polymorphique, les aluminoferrites forment une série de solutions solides de formule $C_{2}\left(A_{1-p} F_{p}\right)$ avec une limite de solubilité des ions $\mathrm{Al}$ atteinte à $\mathrm{p}=0.70$ pour la composition $\mathrm{C}_{2} \mathrm{~F}$. La forme la plus stable est donnée par la brownmillérite $\mathrm{C}_{4} \mathrm{AF}(2)$.

Par ailleurs, il est possible de relier les formes cristallines et leurs propriétés physiques par les illustrations suivantes :

Le silicate tricalcique contribue pour une grande part au durcissement du ciment. Des variations d'activité hydraulique et de résistance, liées à des défauts de structure sont observés aux jeunes âges. Dans ce qu'il 
est convenu de nommer phase interstitielle, l'aluminate tricalcique réagit rapidement avec l'eau et la forme orthorombique contenant des alcalins est plus réactive que la forme cubique aux premiers âges. On constate que $\mathrm{C}_{4} \mathrm{AF}$ a un pouvoir hydraulique faible. Cependant, la réactivité des solutions solides augmente avec la teneur en aluminium.

En résumé, tout cela va se traduire sur les diffractogrammes par de légers déplacements de certaines raies ainsi que par des multiplets serrés et nombreux.

\section{Analyse}

La méthode d'analyse retenue est celle de F.H. CHUNG (3) car elle permet de s'affranchir d'un étalon interne. Elle repose sur la détermination des aires relatives des pics caractéristiques des phases du clinker. La valeur des coefficients entrant dans la base du programme de calcul est obtenue à partir des phases pures de synthèse mélangées en proportions soigneusement définies.

Lorsqu'un faisceau monochromatique de rayons $\mathrm{X}$ arrive sur un échantillon pulvérulent plan, les intensités diffractées sont reliées à la composition par les équations de Klug et Alexander (1959) :

De sorte que :

$$
I_{i}=K_{i} \frac{X_{i} / Q_{i}}{\sum \mu_{i} X_{i}}=K_{i} \frac{X_{i} / Q_{i}}{\mu_{i}}
$$

$$
\mathrm{I}_{\mathrm{t}} / \mathrm{I}_{\mathrm{t}}^{0}=\mathrm{X}_{\mathrm{i}} \frac{\mu_{\mathrm{i}}}{\mu_{\mathrm{t}}}
$$

où :

$\mathrm{I}_{\mathrm{t}}=$ Intensité des rayons $\mathrm{X}$ diffractés par un plan sélectionné (hkl) du composé $\mathrm{i}$

$I_{\mathrm{t}}=$ Intensité des rayons $\mathrm{X}$ diffracté par un plan sélectionné (hkl) du composé pur $\mathrm{i}$

$\mathrm{K}_{\mathrm{t}}=$ Constante qui dépend de la géométrie du diffractomètre et de la nature du composé $\mathrm{i}$

$\mathrm{X}_{\mathrm{i}}=$ Teneur du composé $\mathrm{i}$

$\mathrm{Q}_{\mathrm{i}}=$ Densité du composé $\mathrm{i}$

$\mu_{\mathrm{i}}=$ Coefficient d'absorption massique de l'élément pour $\mathrm{i}$

$\mu_{t}=$ Coefficient d'absorption massique de l'échantillon total exposé au faisceau primaire de rayons $\mathrm{X}$

A partir de ces relations de base, F.H. CHUNG montre que l'on peut relier les rapports d'intensité aux concentrations en s'affranchissant des effets de matrice par la relation suivante:

$$
\mathrm{X}_{\mathrm{i}}=\frac{X_{r}}{K_{i}}=\frac{I_{i}}{I_{r}} \quad \text { avec } \mathrm{K}_{\mathrm{i}}=\frac{I_{i}}{I_{r}}
$$

$\mathrm{I}_{\mathrm{r}}=$ est l'intensité de la phase de référence

Les 4 diffractogrammes qui suivent représentent les phases pures obtenues en laboratoire. La présence de silicium est liée à la nécessité de suivre la synthèse du matériau. Par la suite il n'en est plus fait usage. 


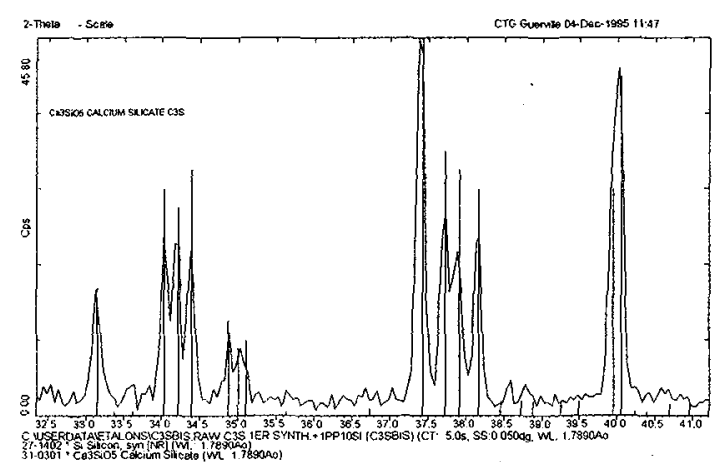

$\mathrm{C} 3 \mathrm{~S}$

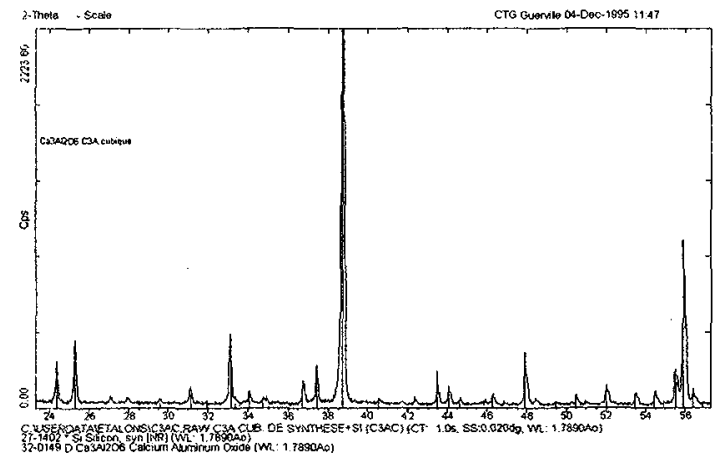

$\mathrm{C} 3 \mathrm{~A}$

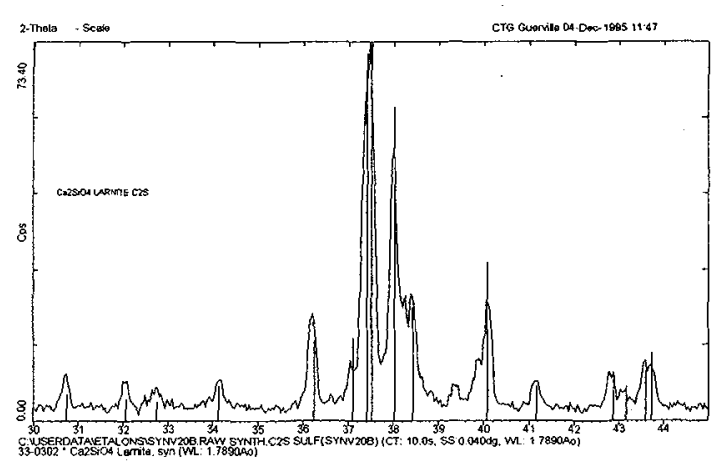

$\mathrm{C} 2 \mathrm{~S}$

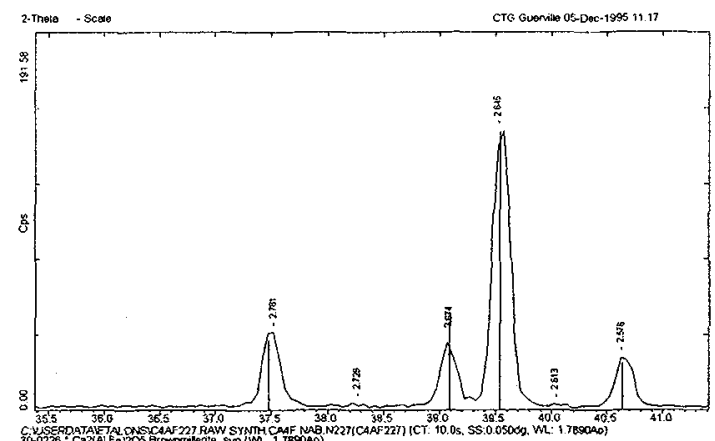

$\mathrm{C} 4 \mathrm{AF}$

L'évaluation quantitative des phases du clinker ne peut se faire qu'en s'affranchissant des interférences indésirables. Pour cela, on procèdera en deux temps : un première mesure se fera sur le clinker tel quel pour mesurer les teneurs en alite et bélite en se servant des rapports suivants :

$$
\begin{aligned}
& \mathbf{I}_{3.03 \mathrm{~A}^{\circ}} \mathrm{ALITE} / \mathbf{I}_{2.88 \mathrm{~A}^{\circ}} \mathrm{BELITE}=\mathrm{K}_{1} \mathbf{C}_{\mathrm{ALITE}} / \mathbf{C}_{\mathrm{BELITE}} \\
& \text { Avec }: \mathbf{C}_{\text {alite }}+\mathbf{C}_{\text {bélite }}=100 \text { - (insoluble salicylique }+\mathrm{CaO} \text { libre) }
\end{aligned}
$$

Les aluminates seront dosés après dissolution sélective des silicates selon une méthode proposée par TAKASHIMA (Japan Cement Engineering Association - 1959).

Les silicates du clinker sont séparés des autres constituants à l'aide d'un solvant approprié constitué d'un mélange d'acide salicylique ( $\mathrm{HO}-\mathrm{C}_{6} \mathrm{H}_{4}-\mathrm{CO}_{2} \mathrm{H}$ acide salicylique ou phénol ortho) et de méthanol.

Des études ont permis de montrer que des mélanges réalisés à partir d'acide maléique conduisent à des résultats analogues.

\section{Mode opératoire}

Le clinker à analyser est broyé jusqu'à refus nul à $40 \mu$. Une prise d'essai de $2 \mathrm{~g}$ est mélangée à $14 \mathrm{~g}$ d'acide salicylique et $75 \mathrm{ml}$ d'alcool méthylique. Le tout est agité pendant 1 heure. 
Après filtration, le filtrat contenant les aluminates et les sulfates est séché à $70^{\circ} \mathrm{C}$ durant 1 heure, pesé puis analysé.

Les pics de diffraction à considérer sur la fraction insoluble salicylique ainsi obtenue se situent dans les domaines angulaires:

$27<2 \Theta<43$ et $47<2 \Theta<53$, avec les $d(\AA)$ correspondant à :

$\mathrm{C}_{3} \mathrm{~A}$ ortho

$\mathrm{C}_{3} \mathrm{~A}$ cubique

Ferrite (brownmillerite)

$\mathrm{SO}_{4} \mathrm{~K}_{2}$ Arcanite

MgO Périclase

Les intensités des
2.67 et 2.65

2.90 et 2.88

$$
\begin{aligned}
& \mathbf{I}_{\left(2.67 \mathrm{~A}^{\circ}+2.65 \mathrm{~A}^{\circ}\right)} \mathrm{FERRITE} / \mathbf{I}_{\left(2.715 \mathrm{~A}^{\circ}+2.695 \mathrm{~A}^{\circ}\right)} \sum \mathrm{C}_{3} \mathrm{~A}=\mathrm{K}_{1} \mathbf{C}_{\mathrm{F}} / \mathbf{C}_{\mathrm{C} 3 \mathrm{~A}} \\
& \mathbf{I}_{\left(2.90 \mathrm{~A}^{\circ}+2.88 \mathrm{~A}^{\circ}\right)} \mathrm{SO}_{4} \mathrm{~K}_{2} / \mathrm{I} \sum \mathrm{C}_{3} \mathrm{~A}=\mathrm{K}_{2} \mathrm{C}_{\text {ARCANITE }} / \mathrm{C}_{\mathrm{C} 3 \mathrm{~A}} \\
& \mathbf{I}_{\left(2.06 \mathrm{~A}^{\circ}\right)} \text { PERICLASE } / \mathrm{I} \sum \mathrm{C}_{3} \mathrm{~A}=\mathrm{K}_{3} \mathrm{C}_{\text {PERICLASE }} / \mathrm{C}_{\mathrm{C} 3 \mathrm{~A}} \\
& \text { Avec : } \mathrm{C}_{3} \mathrm{~A}+\mathrm{FERRITE}+\text { PERICLASE }+ \text { ARCANITE }=100 \\
& \mathrm{~K}_{1}, \mathrm{~K}_{2}, \mathrm{~K}_{3}, \mathrm{~K}_{4} \text { déterminés par la mesure des rapports des aires sur les phases pures. }
\end{aligned}
$$

La contribution du $\mathrm{C}_{3} \mathrm{~A}$ ortho sur le pic à $2.695 \AA ⿱ 乛 ⿻ 上 丨 \mathrm{C}_{3} \mathrm{~A}$ ortho et $\mathrm{C}_{3} \mathrm{~A}$ cub $)=100 / 60$ de l'intensité du pic à $2.72 \AA$ de $\mathrm{C}_{3} \mathrm{~A}$ ortho.

L'intensité de la raie à $2.695 \AA$ de $\mathrm{C}_{3} \mathrm{~A}$ cub seul est identique à la somme des intensités des raies $2.715 \AA$ et $2.695 \AA$ de $\mathrm{C}_{3} \mathrm{~A}$ ortho seul.

(Ces deux hypothèses ont été vérifiées sur des diagrammes de $C_{3} A$ cub et $C_{3} A$ ortho pur).

Ceci suppose l'absence de phases vitreuses dans la fraction insoluble salicylique.

\section{Instrumentation, configuration}

Diffractomètre SIEMENS D500

Détecteur : scintillateur

Tube Co $(\lambda=1.789) 40 \mathrm{KV} 35 \mathrm{~mA}$

Monochromateur avant avec cristal courbe de quartz

Fente réceptrice de $0.15^{\circ}$

Passeur automatique d'échantillons

Acquisition de données par DACO - MP

Traitement des données DIFFRAC AT V3 sur PC

\section{Résultats}

Les diagrammes et les résultats rassemblés sur les pages suivantes illustrent l'utilisation du programme FIT, à partir d'un modèle d'ajustement de courbe (Gauss, Voigt, Pearson ...) sur les phases du clinker tel quel et sur l'insoluble. Le programme FIT fournit la valeur des aires des phases recherchées; elles sont introduites dans un système d'équations à 2 inconnues pour le tel quel et à 4 pour l'insoluble. La comparaison avec les résultats obtenus par fluorescence $\mathrm{X}$ avec laquelle l'analyse élémentaire permet de remonter à la formule potentielle de Bogue indique un écart certain entre les deux méthodes. Il est 
important de signaler que la composition potentielle de Bogue n'est qu'un calcul théorique qui ne tiend pas compte de l'état réel de la clinkérisation et ne correspond pas à une synthèse de laboratoire.

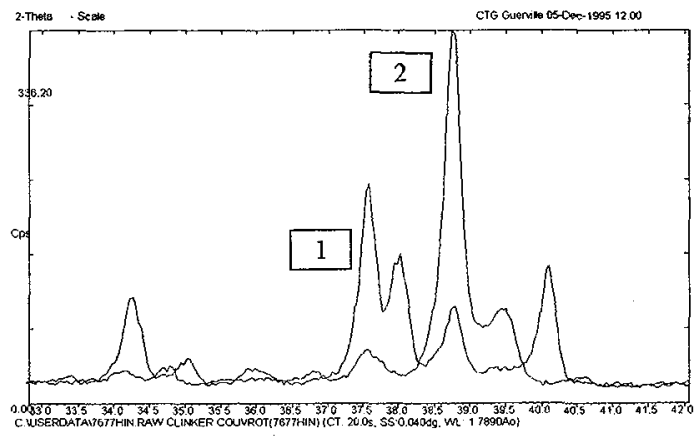

Diagrammes du clinker avant (1) et après (2) dissolution selective.
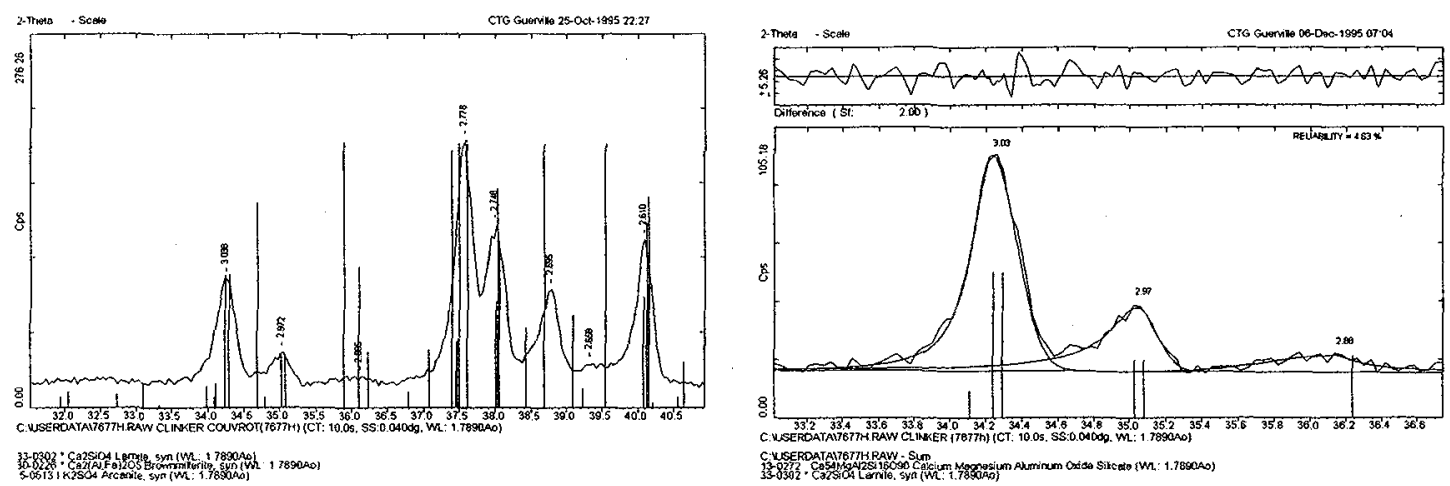

SILICATES

\begin{tabular}{|ll|lc|}
\hline \multicolumn{2}{|c|}{ CHIMIE (FX) \% } & & DRX \% \\
& & & \\
C3S & 62.31 & C3S & 57.6 \\
C2S & 15.86 & C2S & 21.5 \\
& & & \\
\hline
\end{tabular}



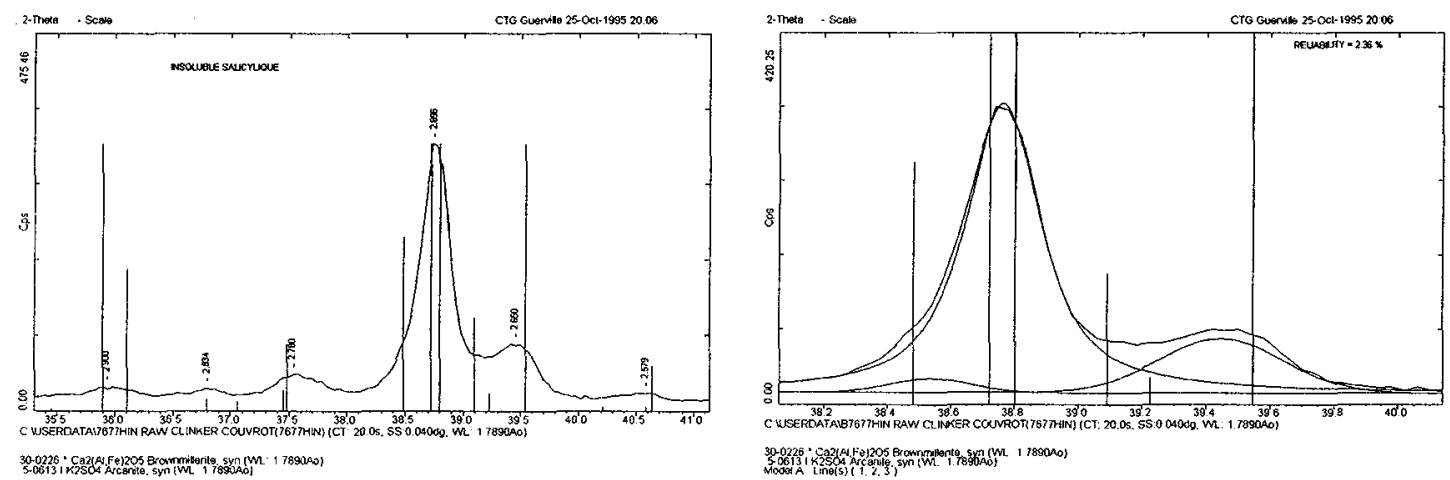

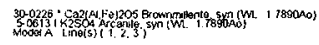

ALUMINATES

\begin{tabular}{|ll|ll|}
\hline \multicolumn{2}{|r|}{ CHIMIE (FX) \% } & \multicolumn{2}{|c|}{ DRX \% } \\
& & C3A Cub & 10.9 \\
C3A & 10.79 & C3A Ortho & 2.0 \\
C4AF & 7.33 & C4AF & 6.5 \\
& & & \\
\hline
\end{tabular}

\section{Conclusions}

Les résultats de diffractométrie obtenus par la méthode utilisée au C.T.G. permettent une quantification des phases du clinker reflétant l'histoire thermique réelle de la synthèse, ce qui n'est pas le cas d'une analyse chimique. Le cimentier, grâce à cet outil et à l'apport de la quantification, est en mesure de remédier à certaines anomalies pouvant se manifester au cours du processus de fabrication.

L'approche actuelle pourra être améliorée par l'intégration de nouvelles méthodes d'affinement de paramètres dont l'usage est grandement facilité part les moyens informatiques.

\section{REFERENCES}

(1) Pliskin L. et coll. La fabrication du ciment. Ciments Français. Eyrolles

(2) Regourd M, Bigare M, Forest $\mathbf{J}$ and Guinier A. Synthesis and cristallographie investigations of some belites Symp.Tokyo 1968

(3) Chung F. H. J. Appl. Cryst. (1974) 7, 519, and J. Appl. Cryst.(1975) 8,17. 